ReVISTA de BIOLOGía TROPICAL

\title{
Testate Amoebae (Amebozoa: Arcellinida) in Tropical Lakes of Central Mexico
}

Itzel Sigala*1, Socorro Lozano-García ${ }^{2}$, Jaime Escobar ${ }^{3,4}$, Liseth Pérez $^{2} \&$ Elvia Gallegos-Neyra 5

1. Posgrado de Ciencias Biológicas, Instituto de Geología, Universidad Nacional Autónoma de México, Ciudad Universitaria, 04510, Distrito Federal, México; itzelsr@yahoo.com.mx

2. Instituto de Geología, Universidad Nacional Autónoma de México, Ciudad Universitaria, 04510, Distrito Federal, México; mslozano@unam.mx,lcpereza@geologia.unam.mx

3. Departamento de Ingeniería Civil y Ambiental, Universidad del Norte, Km 5 Vía Puerto Colombia, Barranquilla, Colombia; jhescobar@uninorte.edu.com

4. Center for Tropical Paleoecology and Archaeology, Smithsonian Tropical Research Institute, Balboa, Ancon, 0843033092, Panamá City, Panamá.

5. Laboratorio de Investigación de Patógenos Emergentes, Unidad de Investigación Interdisciplinaria para las Ciencias de la Salud y Educación, Facultad de Estudios Superiores Iztacala, Universidad Nacional Autónoma de México, Los Reyes Iztacala, 54090, Estado de México, México; elvia.gallegos1@gmail.com

* Correspondence

Received 02-II-2015. C Corrected 20-IX-2015. Accepted 22-X-2015.

\begin{abstract}
Testate amoebae are common single-celled eukaryotic organisms in aquatic ecosystems. Despite their important role in these ecosystems, and their potential as bioindicators and paleoindicators, they remain poorly studied in Mexico. The major objectives of this study were to: 1) increase knowledge of testate amoebae in Mexico's tropical lakes, and 2) create a catalog of high-quality scanning electron micrographs that can be used for future ecological and paleoenvironmental studies. We collected surface-sediment samples from 29 lakes, located in the Transmexican Volcanic Belt, one at each lake during June and October 2011, and March 2013. Sediments were collected with an Ekman grab and preserved in anhydrous ethanol. Sub-samples were observed under a stereomicroscope and morphometric data for each species were recorded. Total diameter and aperture diameter were measured on circular tests. Irregularly shaped tests were measured for length and width of the aperture and for the size of the whole test. If a specimen possessed spines, the length of one randomly selected spine was measured. The best-preserved specimen of each taxon was photographed with an optical microscope and a scanning electron microscope (SEM). We found 41 taxa of testate amoebae belonging to the genera: Arcella, Argynnia, Centropyxis, Cucurbitella, Cyclopyxis, Cyphoderia, Difflugia, Euglypha, Lesquereusia, Pentagonia, Pseudodifflugia and Scutiglypha. Twelve species not previously reported for Mexico were recorded, along with 13 varieties. The average number of taxa recorded in each lake was eight, and the highest taxonomic richness was 18. The taxon found in the greatest number of lakes was Centropyxis aculeata var. aculeata. Taxonomic richness varied among lakes in the same region. This could reflect lake-specific differences in environmental conditions, underscoring the need for more detailed studies that include collection of data on physical and chemical variables in the lakes. Our results highlighted the need of further studies for the distribution patterns and ecology of lacustrine testate amoebae. Rev. Biol. Trop. 64 (1): 377-397. Epub 2016 March 01.
\end{abstract}

Key words: protozoa, amoeboid, thecamoebians, taxonomy, biometry.

Testate amoebae are found in almost all freshwater habitats. They are present in springs, lakes, reservoirs, waterfalls, rivers, cenotes and even subterranean pools (Medioli \& Scott, 1988; Patterson \& Kumar, 2002; Escobar, Martínez, \& Parra, 2005; van Hengstum,
Reinhardt, Beddows, Huang, \& Gabriel, 2008). These organisms are a diverse and important component of the benthic community in lakes and wetlands and they play a critical role in food webs (Neville, Christie, McCarthy, \& MacKinnon, 2010). 
Also referred to as thecamoebians, testate amoebae are single-celled amoeboid protozoa in which the cytoplasm is enclosed within an external shell or discrete test from which pseudopodia emerge (Ogden \& Hedley, 1980). These tests range in size from 5 to $300 \mu \mathrm{m}$ (Smith, Bobrov, \& Lara, 2008). Some species have an autogenous test that can be proteinaceous, siliceous or, rarely, calcareous. Other species possess a xenogenous test formed of agglutinated organic or mineral particles captured from the surrounding environment (Ogden \& Hedley, 1980; Meisterfeld, 2002a, 2002b). Test morphologies are diverse, which allows identification of these organisms to the species level (Mitchell, Charman, \& Warner, 2008).

We studied a database of $\sim 2800$ publications on testate amoebae worldwide (Medioli, Bonnet, Scott, \& Medioli, 2003). Results showed that the majority of studies were conducted in temperate, Northern Hemisphere countries. Nevertheless, tropical countries such as Mexico, Brazil, the Democratic Republic of the Congo, Republic of the Congo and India had a few publications on testate amoebae over the last century. The greatest numbers of studies worldwide were of taxonomic nature, and a few in tropical localities were related to ecology or paleoecology. More than the half of the research accomplished in tropical latitudes was conformed by technical publications or unpublished reports. There is thus a knowledge gap regarding thecamoebians in tropical countries, and further studies are needed in these regions. Additionally, most previous taxonomic research in the Neotropics identified species using only light microscopy (Lahr \& Lopes, 2006). The increasing use of testate amoebae as bioindicators and paleoindicators has increased the need for further studies to clarify taxonomy, and elucidate distribution patterns and ecology.

Published studies from Mexico focused on testate amoebae in soils and mosses of diverse terrestrial ecosystems (Golemansky, 1967; Bonnet, 1977; Bobrov \& Krasil'nikov, 2011; Bobrov, Krasil'nikov, \& García-Calderón, 2013; Gallegos-Neyra, Lugo-Vázquez,
Calderón-Vega, Sánchez-Rodríguez, \& Mayén-Estrada, 2014). There is also information from protozoological studies focused on lakes (López-Ochoterena \& Roure-Cane 1970; Aladro, Reyes, Olvera, \& Robles, 2007), cenotes (van Hengstum et al., 2008) and caves (Osorio-Tafall, 1943; Hoffmann, Palacios, \& Morales-Malacara, 1986).

The goals of this study were to identify species assemblages of testate amoebae from lakes distributed across central Mexico, in the Northern Neotropics, and produce a catalog of high-quality scanning electron microscope images of those taxa that can be used for future ecological and paleoenvironmental studies.

\section{MATERIALS AND METHODS}

Samples were collected from 29 lakes across central Mexico in June and October 2011 and March 2013 (Table 1). The lakes are located within the Neotropical Transmexican Volcanic Belt (Fig. 1). Surface sediment samples were collected with an Ekman grab at the deepest site in each lake (Table 1) and at littoral sites, 0.5 to $1 \mathrm{~m}$ deep. In the laboratory, sediment samples were preserved in anhydrous ethanol and sub-samples of $2 \mathrm{~cm}^{3}$ wet volume were examined in a Petri dish under a stereomicroscope (Zeiss STEMI 2000-C Schott SeriesEasyLED). Testate amoebae tests in each sediment sub-sample were extracted using a fine brush (Ellison \& Ogden, 1987).

Total diameter and aperture diameter were measured on circular tests. Irregularly shaped tests were measured for length and width of the aperture and for the size of the whole test. If a specimen possessed spines, the length of one randomly selected spine was measured. Morphometric data were recorded on at least 30 specimens of each species, with preference given to specimens from the same lake. In some cases, however, there were insufficient tests to measure the required 30 specimens from a single lake, in which case we used individuals from different lakes. In the event that the total number of specimens of a taxon, even from multiple sample lakes, failed to yield 


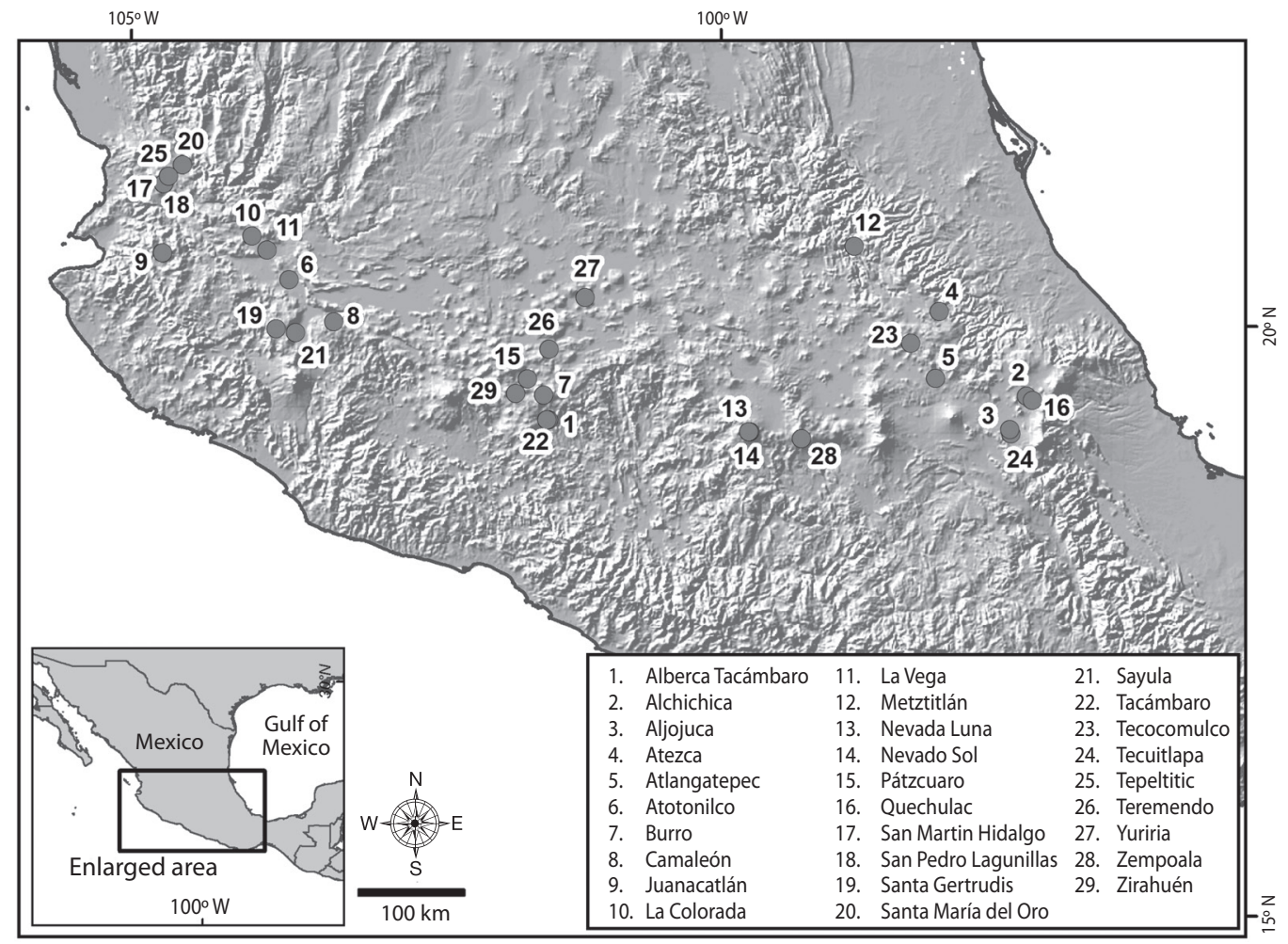

Fig. 1. Location of the 29 study lakes in Mexico.

enough tests, we simply measured those we had collected.

The best-preserved specimen of each taxon was photographed. Photographs were taken with an Olympus BX50 optical microscope and Olympus DP11 camera, and a Jeol JSM 5 600-LV LCM scanning electron microscope (SEM). Identification was made using available taxonomic keys for testate amoebae, including Ogden and Hedley (1980), Kumar and Dalby (1998), Lee, Leedale and Bradbury (2000), Charman, Hendon and Woodland (2000) and Escobar, Brenner, Whitmore, Kenney and Curtis (2008). Lacustrine arcellacean species can display a large amount of ecophenotypically controlled morphological variability. It is accepted practice that researchers working in lakes assign informal infrasubspecific 'strain' names for ecophenotypes to avoid possible description of unwarranted new species. Although the International Code of Zoological Nomenclature stipulates that infra-subspecfic-level designations have no status (International Commission on Zoological Nomenclature [ICZN], 1999), they are useful for distinguishing environmentally significant populations in lacustrine environments (Patterson, Roe, \& Swindles, 2012).

\section{RESULTS}

Fifty-three surface-sediment samples from 29 lakes were examined for testate amoebae. In total, we identified 41 taxa (24 species and 17 varieties) that belonged to 12 genera (Arcella, Argynnia, Centropyxis, Cucurbitella, Cyclopyxis, Cyphoderia, Difflugia, Euglypha, Lesquereusia, Pentagonia, Pseudodifflugia and Scutiglypha). Of those, 12 species (Arcella conica, Argynnia triangulata, Centropyxis deflandrei, Cucurbitella tricuspis, Difflugia labiosa, D. distenda, D. bidens, D. fragosa, 
TABLE 1

Geographic coordinates and morphometric variables for the study lakes

\begin{tabular}{|c|c|c|c|c|c|c|}
\hline Lake Number & Lake Name & Latitude $(\mathrm{N})$ & Longitude (W) & Altitude (masl) & Area (ha) & Maximum Depth (m) \\
\hline 1 & Alberca Tacámbaro & $19^{\circ} 12^{\prime} 38^{\prime \prime}$ & $101^{\circ} 27^{\prime} 33^{\prime \prime}$ & 1475 & 7.7 & 33.0 \\
\hline 2 & Alchichica & $19^{\circ} 24^{\prime} 44^{\prime \prime}$ & $97^{\circ} 24^{\prime} 7^{\prime \prime}$ & 2321 & 200.0 & 64.0 \\
\hline 3 & Aljojuca & $19^{\circ} 5 ' 23^{\prime \prime}$ & $97^{\circ} 32^{\prime} 5^{\prime \prime}$ & 2371 & 42.0 & 51.0 \\
\hline 4 & Atezca & $20^{\circ} 7^{\prime} 46^{\prime \prime}$ & $98^{\circ} 8^{\prime} 39^{\prime \prime}$ & 1316 & 26.7 & 15.0 \\
\hline 5 & Atlangatepec & 19॰33'35' & $98^{\circ} 10^{\prime} 38^{\prime \prime}$ & 2511 & 755.0 & 1.2 \\
\hline 6 & Atotonilco & $20^{\circ} 23^{\prime} 58^{\prime \prime}$ & $103^{\circ} 39^{\prime} 47^{\prime \prime}$ & 1355 & 1370.0 & 0.5 \\
\hline 7 & Burro & $19^{\circ} 25^{\prime} 7^{\prime \prime}$ & $101^{\circ} 30^{\prime} 9^{\prime \prime}$ & 2708 & 9.0 & 1.0 \\
\hline 8 & Camaleón & $20^{\circ} 27^{\prime \prime}$ & $103^{\circ} 16^{\prime} 58^{\prime \prime}$ & 1879 & 1.3 & 1.8 \\
\hline 9 & Juanacatlán & $20^{\circ} 37^{\prime} 39^{\prime \prime}$ & $104^{\circ} 44^{\prime} 17^{\prime \prime}$ & 1981 & 20.0 & 25.0 \\
\hline 10 & La Colorada & $20^{\circ} 45^{\prime} 56^{\prime \prime}$ & $103^{\circ} 58^{\prime} 49^{\prime \prime}$ & 1366 & 410.0 & 2.8 \\
\hline 11 & La Vega & $20^{\circ} 38^{\prime} 49^{\prime \prime}$ & $103^{\circ} 51^{\prime} 9^{\prime \prime}$ & 1274 & 1610.0 & 2.4 \\
\hline 12 & Metztitlán & $20^{\circ} 40^{\prime} 53^{\prime \prime}$ & $98^{\circ} 51^{\prime} 56^{\prime \prime}$ & 1258 & 2940.0 & 3.0 \\
\hline 13 & Nevado Luna & $19^{\circ} 64^{\prime \prime}$ & $99^{\circ} 45^{\prime} 9^{\prime \prime}$ & 4283 & 2.5 & 10.0 \\
\hline 14 & Nevado Sol & $19^{\circ} 69^{\prime \prime}$ & $99^{\circ} 45^{\prime} 34^{\prime \prime}$ & 4283 & 17.5 & 15.0 \\
\hline 15 & Pátzcuaro & $19^{\circ} 33^{\prime} 18^{\prime \prime}$ & $101^{\circ} 38^{\prime} 17^{\prime \prime}$ & 2041 & 10000.0 & 11.0 \\
\hline 16 & Quechulac & $19^{\circ} 22^{\prime} 28^{\prime \prime}$ & $97^{\circ} 21^{\prime} 6 "$ & 2345 & 64.0 & 40.0 \\
\hline 17 & San Martín Hidalgo & $21^{\circ} 12^{\prime} 33^{\prime \prime}$ & $104^{\circ} 43^{\prime} 37^{\prime \prime}$ & 1261 & 296.0 & 7.0 \\
\hline 18 & San Pedro Lagunillas & $21^{\circ} 12^{\prime} 33^{\prime \prime}$ & $104^{\circ} 43^{\prime} 37^{\prime \prime}$ & 1261 & 296.0 & 7.0 \\
\hline 19 & Santa Gertrudis & $19^{\circ} 58^{\prime} 57^{\prime \prime}$ & $103^{\circ} 46^{\prime} 20^{\prime \prime}$ & 1743 & 50.0 & 2.3 \\
\hline 20 & Santa María del Oro & $21^{\circ} 22^{\prime} 10^{\prime \prime}$ & $104^{\circ} 34^{\prime} 9^{\prime \prime}$ & 737 & 370.0 & 65.0 \\
\hline 21 & Sayula & $19^{\circ} 57^{\prime} 4^{\prime \prime}$ & $103^{\circ} 36^{\prime} 33^{\prime \prime}$ & 1347 & 10700.0 & 1.0 \\
\hline 22 & Tacámbaro & $19^{\circ} 12^{\prime} 30^{\prime \prime}$ & $101^{\circ} 28^{\prime} 22^{\prime \prime}$ & 1517 & 21.5 & 4.0 \\
\hline 23 & Tecocomulco & $19^{\circ} 51^{\prime} 37^{\prime \prime}$ & $98^{\circ} 23^{\prime} 13^{\prime \prime}$ & 2535 & 1900.0 & 2.0 \\
\hline 24 & Tecuitlapa & $19^{\circ} 7^{\prime} 30^{\prime \prime}$ & $97^{\circ} 32^{\prime} 36^{\prime \prime}$ & 2368 & 18.0 & 1.5 \\
\hline 25 & Tepeltitic & $21^{\circ} 16^{\prime} 30^{\prime \prime}$ & $104^{\circ} 41^{\prime} 18^{\prime \prime}$ & 1430 & 132.0 & 2.5 \\
\hline 26 & Teremendo & $19^{\circ} 48^{\prime} 21^{\prime \prime}$ & $101^{\circ} 27^{\prime} 15^{\prime \prime}$ & 2058 & 15.0 & 9.0 \\
\hline 27 & Yuriria & $20^{\circ} 14^{\prime} 51^{\prime \prime}$ & $101^{\circ} 8^{\prime} 58^{\prime \prime}$ & 1730 & 5000.0 & 2.3 \\
\hline 28 & Zempoala & $19^{\circ} 3^{\prime} 0^{\prime \prime}$ & $99^{\circ} 18^{\prime} 50^{\prime \prime}$ & 2804 & 10.0 & 6.0 \\
\hline 29 & Zirahuén & $19^{\circ} 26^{\prime} 7^{\prime \prime}$ & $101^{\circ} 44^{\prime} 22^{\prime \prime}$ & 2082 & 930.0 & 40.0 \\
\hline
\end{tabular}

Lesquereusia spiralis, Pentagonia maroccana, Pseudodifflugia fulva and Scutiglypha cabrolae) and 13 varieties (Centropyxis constricta var. spinosa, Difflugia oblonga var. bryophila, D. oblonga var. glans, D. oblonga var. lanceolata, D. oblonga var. linearis, D. oblonga var. oblonga, D. oblonga var. spinosa, D. oblonga var. tenuis, D. protaeiformis var. acuminata, D. protaeiformis var. amphoralis, D. protaeiformis var. claviformis, D. urceolata var. elongata, D. urceolata var. urceolata) had not been recorded previously in Mexico. Scanning electron microscope photographs were taken from the main taxa for better identification (Fig. 2, Fig. 3 y Fig. 4) and some details of the structure of their tests were also recorded using higher magnification (Fig. 5). Morphometric data for all taxa are summarized in Table 2.

\section{Species characterization and previous occurrences}

Arcella conica Deflandre, 1928

Autogenous brown test. Hexagonal in ventral view with a central, circular aperture. Domed in lateral and dorsal view with 
TABLE 2

Morphologic data for each taxon found in the lakes

\begin{tabular}{|c|c|c|c|c|c|c|}
\hline Species/Character & Mean $(\mu \mathrm{m})$ & $\mathrm{SD}$ & $\mathrm{CV}$ & $\operatorname{Min}(\mu \mathrm{m})$ & $\operatorname{Max}(\mu \mathrm{m})$ & $\mathrm{n}$ \\
\hline \multicolumn{7}{|l|}{ Arcella conica } \\
\hline td & 100.0 & - & - & - & - & 1 \\
\hline ad & 30.0 & - & - & - & - & 1 \\
\hline \multicolumn{7}{|l|}{ Arcella dentata } \\
\hline td & 144.3 & 36.7 & 0.3 & 68.0 & 176.0 & 6 \\
\hline $\mathrm{ad}$ & 32.3 & 7.3 & 0.2 & 24.0 & 42.0 & 6 \\
\hline \multicolumn{7}{|l|}{ Arcella discoides } \\
\hline td & 131.2 & 45.4 & 0.4 & 50.0 & 250.0 & 31 \\
\hline ad & 59.6 & 26.7 & 0.5 & 18.0 & 114.0 & 31 \\
\hline \multicolumn{7}{|l|}{ Arcella gibbosa } \\
\hline td & 42.0 & - & - & - & - & 1 \\
\hline $\mathrm{ad}$ & 12.0 & - & - & - & - & 1 \\
\hline \multicolumn{7}{|l|}{ Arcella megastoma } \\
\hline td & 180.7 & 55.9 & 0.3 & 84.0 & 360.0 & 20 \\
\hline ad & 97.7 & 33.6 & 0.3 & 50.0 & 200.0 & 19 \\
\hline \multicolumn{7}{|l|}{ Arcella vulgaris } \\
\hline td & 104.8 & 25.2 & 0.2 & 42.0 & 148.0 & 31 \\
\hline ad & 27.7 & 10.7 & 0.4 & 10.0 & 68.0 & 30 \\
\hline \multicolumn{7}{|l|}{ Argynnia triangulata } \\
\hline $\mathrm{tl}$ & 168.8 & 26.5 & 0.2 & 150.0 & 187.0 & 2 \\
\hline tw & 155.0 & 21.2 & 0.1 & 140.0 & 170.0 & 2 \\
\hline ad & 80.8 & 9.6 & 0.1 & 74.0 & 87.5 & 2 \\
\hline \multicolumn{7}{|l|}{ Centropyxis aculeata var. aculeata } \\
\hline $\mathrm{tl}$ & 131.7 & 24.8 & 0.2 & 79.0 & 180.0 & 40 \\
\hline tw & 131.3 & 31.4 & 0.2 & 24.0 & 180.0 & 38 \\
\hline al & 45.7 & 11.2 & 0.3 & 22.0 & 80.0 & 40 \\
\hline$a b$ & 47.6 & 11.8 & 0.3 & 21.0 & 75.0 & 38 \\
\hline sl & 24.3 & 7.6 & 0.3 & 8.0 & 46.0 & 38 \\
\hline \multicolumn{7}{|l|}{ Centropyxis aculeata var. discoides } \\
\hline $\mathrm{tl}$ & 123.3 & 20.5 & 0.2 & 90.0 & 187.5 & 31 \\
\hline tw & 119.9 & 21.9 & 0.2 & 90.0 & 184.5 & 31 \\
\hline al & 42.1 & 9.0 & 0.2 & 24.0 & 67.5 & 31 \\
\hline$a b$ & 45.2 & 9.8 & 0.2 & 28.0 & 64.0 & 31 \\
\hline sl & 17.1 & 4.4 & 0.3 & 10.0 & 24.0 & 9 \\
\hline \multicolumn{7}{|c|}{ Centropyxis constricta var. aerophila } \\
\hline $\mathrm{tl}$ & 76.8 & 32.8 & 0.4 & 50.0 & 124.0 & 5 \\
\hline tw & 53.8 & 29.7 & 0.6 & 22.0 & 88.0 & 5 \\
\hline al & 26.6 & 8.2 & 0.3 & 20.0 & 36.0 & 5 \\
\hline$a b$ & 31.0 & 17.6 & 0.6 & 16.0 & 56.0 & 5 \\
\hline \multicolumn{7}{|c|}{ Centropyxis constricta var. constricta } \\
\hline $\mathrm{tl}$ & 76.0 & - & - & - & - & 1 \\
\hline tw & 78.0 & - & - & - & - & 1 \\
\hline al & 34.0 & - & - & - & - & 1 \\
\hline$a b$ & 40.0 & - & - & - & - & 1 \\
\hline sl & 14.0 & - & - & - & - & 1 \\
\hline
\end{tabular}


TABLE 2 (Continued)

\begin{tabular}{|c|c|c|c|c|c|c|}
\hline Species/Character & Mean $(\mu \mathrm{m})$ & SD & $\mathrm{CV}$ & $\operatorname{Min}(\mu \mathrm{m})$ & $\operatorname{Max}(\mu \mathrm{m})$ & $\mathrm{n}$ \\
\hline \multicolumn{7}{|l|}{ Centropyxis constricta var. spinosa } \\
\hline $\mathrm{tl}$ & 83.1 & 14.2 & 0.2 & 44.8 & 120.0 & 30 \\
\hline tw & 77.2 & 12.1 & 0.2 & 42.6 & 106.0 & 30 \\
\hline al & 32.7 & 7.1 & 0.2 & 16.8 & 48.0 & 30 \\
\hline$a b$ & 38.4 & 7.4 & 0.2 & 16.8 & 54.0 & 30 \\
\hline sl & 18.0 & 5.1 & 0.3 & 8.0 & 28.0 & 24 \\
\hline \multicolumn{7}{|l|}{ Centropyxis deflandrei } \\
\hline $\mathrm{tl}$ & 106.0 & - & - & - & - & 1 \\
\hline tw & 94.0 & - & - & - & - & 1 \\
\hline al & 48.0 & - & - & - & - & 1 \\
\hline$a b$ & 48.0 & - & - & - & - & 1 \\
\hline \multicolumn{7}{|l|}{ Centropyxis ecornis } \\
\hline $\mathrm{tl}$ & 168.0 & - & - & - & - & 1 \\
\hline tw & 156.0 & - & - & - & - & 1 \\
\hline al & 62.0 & - & - & - & - & 1 \\
\hline$a b$ & 60.0 & - & - & - & - & 1 \\
\hline \multicolumn{7}{|l|}{ Cucurbitella tricuspis } \\
\hline $\mathrm{tl}$ & 109.9 & 32.7 & 0.3 & 48.8 & 170.0 & 30 \\
\hline tw & 102.5 & 30.5 & 0.3 & 51.0 & 152.0 & 30 \\
\hline ad & 39.6 & 12.9 & 0.3 & 8.0 & 60.0 & 30 \\
\hline \multicolumn{7}{|l|}{ Cyclopyxis kahli } \\
\hline tw & 87.2 & 28.3 & 0.3 & 50.0 & 130.0 & 6 \\
\hline ad & 27.8 & 17.8 & 0.6 & 12.0 & 62.0 & 6 \\
\hline \multicolumn{7}{|l|}{ Cyphoderia ampulla } \\
\hline tl & 407.5 & - & - & - & - & 1 \\
\hline tw & 162.5 & - & - & - & - & 1 \\
\hline $\mathrm{ad}$ & 25.0 & - & - & - & - & 1 \\
\hline \multicolumn{7}{|l|}{ Difflugia labiosa } \\
\hline $\mathrm{tl}$ & 93.0 & 4.2 & 0.1 & 90.0 & 96.0 & 2 \\
\hline tw & 62.0 & 11.3 & 0.2 & 54.0 & 70.0 & 2 \\
\hline $\mathrm{ad}$ & 26.0 & 5.7 & 0.2 & 22.0 & 30.0 & 2 \\
\hline \multicolumn{7}{|l|}{ Difflugia distenda } \\
\hline $\mathrm{tl}$ & 140.0 & - & - & - & - & 1 \\
\hline tw & 120.0 & - & - & - & - & 1 \\
\hline ad & 68.0 & - & - & - & - & 1 \\
\hline sl & 30.0 & - & - & - & - & 1 \\
\hline \multicolumn{7}{|l|}{ Difflugia bidens } \\
\hline tl & 110.4 & 18.1 & 0.2 & 64.0 & 162.5 & 29 \\
\hline tw & 106.1 & 15.3 & 0.1 & 50.0 & 137.5 & 29 \\
\hline $\mathrm{ad}$ & 51.4 & 11.4 & 0.2 & 26.0 & 75.0 & 29 \\
\hline sl & 15.4 & 4.6 & 0.3 & 8.0 & 24.0 & 28 \\
\hline \multicolumn{7}{|l|}{ Difflugia corona } \\
\hline $\mathrm{tl}$ & 115.3 & 14.2 & 0.1 & 86.0 & 154.0 & 30 \\
\hline tw & 106.7 & 15.4 & 0.1 & 80.0 & 144.0 & 30 \\
\hline ad & 44.7 & 10.3 & 0.2 & 24.0 & 72.0 & 30 \\
\hline sl & 17.9 & 7.7 & 0.4 & 8.0 & 36.0 & 24 \\
\hline
\end{tabular}


TABLE 2 (Continued)

\begin{tabular}{|c|c|c|c|c|c|c|}
\hline Species/Character & Mean $(\mu \mathrm{m})$ & $\mathrm{SD}$ & $\mathrm{CV}$ & $\operatorname{Min}(\mu \mathrm{m})$ & $\operatorname{Max}(\mu \mathrm{m})$ & $\mathrm{n}$ \\
\hline \multicolumn{7}{|l|}{ Difflugia fragosa } \\
\hline $\mathrm{tl}$ & 132.0 & 19.8 & 0.2 & 118.0 & 146.0 & 2 \\
\hline tw & 103.0 & 7.1 & 0.1 & 98.0 & 108.0 & 2 \\
\hline $\mathrm{ad}$ & 45.0 & 4.2 & 0.1 & 42.0 & 48.0 & 2 \\
\hline \multicolumn{7}{|l|}{ Difflugia gramen } \\
\hline $\mathrm{tl}$ & 75.3 & 16.9 & 0.2 & 50.0 & 120.0 & 30 \\
\hline tw & 57.3 & 13.3 & 0.2 & 40.0 & 80.0 & 30 \\
\hline ad & 23.9 & 7.7 & 0.3 & 12.0 & 40.0 & 30 \\
\hline \multicolumn{7}{|l|}{ Difflugia oblonga var. bryophila } \\
\hline tl & 271.8 & 40.2 & 0.2 & 112.0 & 315.0 & 30 \\
\hline tw & 153.4 & 26.4 & 0.2 & 72.0 & 180.0 & 30 \\
\hline ad & 81.2 & 18.0 & 0.2 & 34.0 & 110.0 & 30 \\
\hline \multicolumn{7}{|l|}{ Difflugia oblonga var. glans } \\
\hline tl & 84.0 & 37.4 & 0.5 & 50.0 & 220.0 & 16 \\
\hline tw & 61.1 & 30.1 & 0.5 & 34.0 & 170.0 & 16 \\
\hline ad & 26.6 & 17.6 & 0.7 & 14.0 & 90.0 & 16 \\
\hline \multicolumn{7}{|l|}{ Difflugia oblonga var. lanceolata } \\
\hline tl & 140.0 & - & - & - & - & 1 \\
\hline tw & 62.0 & - & - & - & - & 1 \\
\hline $\mathrm{ad}$ & 28.0 & - & - & - & - & 1 \\
\hline \multicolumn{7}{|l|}{ Difflugia oblonga var. linearis } \\
\hline $\mathrm{tl}$ & 134.1 & 22.3 & 0.2 & 100.0 & 174.0 & 28 \\
\hline tw & 53.6 & 10.9 & 0.2 & 38.0 & 80.0 & 28 \\
\hline $\mathrm{ad}$ & 27.4 & 5.3 & 0.2 & 16.0 & 34.0 & 28 \\
\hline \multicolumn{7}{|l|}{ Difflugia oblonga var. oblonga } \\
\hline $\mathrm{tl}$ & 144.8 & 36.8 & 0.3 & 92.0 & 240.0 & 16 \\
\hline tw & 85.8 & 29.0 & 0.3 & 56.0 & 180.0 & 16 \\
\hline $\mathrm{ad}$ & 44.1 & 23.0 & 0.5 & 12.0 & 118.0 & 16 \\
\hline \multicolumn{7}{|l|}{ Difflugia oblonga var. spinosa } \\
\hline $\mathrm{tl}$ & 186.8 & 74.0 & 0.4 & 96.0 & 296.0 & 5 \\
\hline tw & 106.8 & 31.6 & 0.3 & 64.0 & 146.0 & 5 \\
\hline ad & 38.8 & 9.7 & 0.3 & 28.0 & 50.0 & 5 \\
\hline \multicolumn{7}{|l|}{ Difflugia oblonga var. tenuis } \\
\hline $\mathrm{tl}$ & 226.7 & 89.5 & 0.4 & 126.0 & 370.0 & 15 \\
\hline tw & 117.1 & 52.8 & 0.5 & 58.0 & 195.0 & 15 \\
\hline $\mathrm{ad}$ & 48.5 & 24.7 & 0.5 & 22.0 & 90.0 & 15 \\
\hline \multicolumn{7}{|c|}{ Difflugia protaeiformis var. acuminata } \\
\hline $\mathrm{tl}$ & 192.2 & 23.1 & 0.1 & 148.0 & 258.0 & 30 \\
\hline tw & 68.0 & 16.0 & 0.2 & 28.0 & 130.0 & 30 \\
\hline ad & 40.6 & 6.4 & 0.2 & 26.0 & 60.0 & 30 \\
\hline \multicolumn{7}{|c|}{ Difflugia protaeiformis var. amphoralis } \\
\hline $\mathrm{tl}$ & 95.3 & 40.0 & 0.4 & 56.0 & 210.0 & 31 \\
\hline tw & 61.6 & 24.8 & 0.4 & 38.0 & 140.0 & 31 \\
\hline $\mathrm{ad}$ & 28.8 & 13.0 & 0.5 & 16.0 & 74.0 & 30 \\
\hline \multicolumn{7}{|c|}{ Difflugia protaeiformis var. claviformis } \\
\hline $\mathrm{tl}$ & 160.0 & - & - & - & - & 1 \\
\hline tw & 64.0 & - & - & - & - & 1 \\
\hline ad & 36.0 & - & - & - & - & 1 \\
\hline
\end{tabular}


TABLE 2 (Continued)

\begin{tabular}{|c|c|c|c|c|c|c|}
\hline Species/Character & Mean $(\mu \mathrm{m})$ & SD & $\mathrm{CV}$ & $\operatorname{Min}(\mu \mathrm{m})$ & $\operatorname{Max}(\mu \mathrm{m})$ & $\mathrm{n}$ \\
\hline \multicolumn{7}{|l|}{ Difflugia urceolata var. elongata } \\
\hline $\mathrm{tl}$ & 74.7 & 28.6 & 0.4 & 44.0 & 150.0 & 28 \\
\hline tw & 55.2 & 23.9 & 0.4 & 26.0 & 124.0 & 28 \\
\hline $\mathrm{ad}$ & 24.9 & 11.5 & 0.5 & 10.0 & 66.0 & 28 \\
\hline \multicolumn{7}{|l|}{ Difflugia urceolata var. urceolata } \\
\hline tl & 177.1 & 16.6 & 0.1 & 136.0 & 206.0 & 21 \\
\hline tw & 159.8 & 16.4 & 0.1 & 130.0 & 194.0 & 21 \\
\hline ad & 124.1 & 24.8 & 0.2 & 60.0 & 172.0 & 21 \\
\hline \multicolumn{7}{|l|}{ Euglypha acanthophora } \\
\hline $\mathrm{tl}$ & 110.5 & 50.2 & 0.5 & 75.0 & 146.0 & 2 \\
\hline tw & 67.5 & 37.5 & 0.6 & 41.0 & 94.0 & 2 \\
\hline ad & 25.0 & 12.7 & 0.5 & 16.0 & 34.0 & 2 \\
\hline \multicolumn{7}{|l|}{ Lesquereusia modesta } \\
\hline $\mathrm{tl}$ & 115.3 & 9.9 & 0.1 & 104.0 & 122.0 & 3 \\
\hline tw & 85.3 & 4.2 & 0.1 & 82.0 & 90.0 & 3 \\
\hline $\mathrm{ad}$ & 27.3 & 6.4 & 0.2 & 20.0 & 32.0 & 3 \\
\hline \multicolumn{7}{|l|}{ Lesquereusia spiralis } \\
\hline $\mathrm{tl}$ & 150.0 & - & - & - & - & 1 \\
\hline tw & 120.0 & - & - & - & - & 1 \\
\hline ad & 40.0 & - & - & - & - & 1 \\
\hline sl & 18.0 & - & - & - & - & 1 \\
\hline \multicolumn{7}{|l|}{ Pentagonia maroccana } \\
\hline tl & 90.0 & 8.7 & 0.1 & 80.0 & 100.0 & 5 \\
\hline tw & 52.0 & 4.7 & 0.1 & 48.0 & 60.0 & 5 \\
\hline ad & 27.6 & 1.7 & 0.1 & 26.0 & 30.0 & 5 \\
\hline \multicolumn{7}{|l|}{ Pseudodifflugia fulva } \\
\hline tl & 83.8 & 9.5 & 0.1 & 56.0 & 96.0 & 26 \\
\hline tw & 84.8 & 11.6 & 0.1 & 62.0 & 106.0 & 26 \\
\hline al & 31.3 & 7.3 & 0.2 & 16.0 & 40.0 & 18 \\
\hline $\mathrm{ab}$ & 39.3 & 5.8 & 0.2 & 26.0 & 48.0 & 18 \\
\hline sl & 18.1 & 5.2 & 0.3 & 12.0 & 32.0 & 17 \\
\hline \multicolumn{7}{|l|}{ Scutiglypha cabrolae } \\
\hline $\mathrm{tl}$ & 82.0 & - & - & - & - & 1 \\
\hline tw & 54.0 & - & - & - & - & 1 \\
\hline ad & 34.0 & - & - & - & - & 1 \\
\hline sl & 22.0 & - & - & - & - & 1 \\
\hline
\end{tabular}

All measurements are in $\mu \mathrm{m} . \mathbf{a b}=$ aperture width, $\mathbf{a d}=$ aperture diameter, $\mathbf{a l}=$ aperture length, $\mathbf{t l}=$ test length, $\mathbf{t w}=$ test width, $\mathbf{t d}=$ test diameter, $\mathbf{s} \mathbf{I}=$ spine length. Mean = arithmetic mean, $\mathbf{S D}=$ standard deviations; $\mathbf{C V}=$ coefficient of variation; $\mathbf{M i n}=$ minimum; $\mathbf{M a x}=$ maximum; $\mathbf{n}=$ number of tests. 
depressions forming angular facets bordered by prominent folds and flattened vertices (Fig. 2A). This is the first record of this species in Mexico.

\section{Arcella dentata Ehrenberg, 1830}

Autogenous colorless or yellow test. Circular in ventral view, with 9 to 14 spine-like projections that either formed incipiently or were well developed. Aperture: circular and central. Shallow-bowl shaped in lateral view with flattened vertex and 9 to 14 ridges in dorsal view (Fig. 2B, Fig. 2C, Fig. 5A). The species was observed previously in Mexico in a semipermanent freshwater body (Tiscareño, 2008).

\section{Arcella discoides Ehrenberg, 1843}

Autogenous test, colorless, yellow, brown, white, red or black. Circular in ventral view with a smooth surface. The aperture is circular, invaginated and bordered by a shallow lip, usually surrounded by a ring of numerous small pores. Plano-convex in lateral view, sometimes arched with a basal border (Fig. 2D, Fig. 2E, Fig. 5B). The species was previously observed in Mexico in lakes, springs and wetlands (López-Ochoterena \& Roure-Cane 1970; Bobrov et al., 2013).

\section{Arcella gibbosa Penard, 1890}

Autogenous yellow test. Circular in ventral view. Test surface is irregular with numerous small pores between the circular plates. Aperture invaginated, circular and with a distinct lip. Hemispherical in lateral view, with regular depressions on the dorsal surface (Fig. 2F, Fig. $2 \mathrm{G}$, Fig. 5C). The species was observed previously in Mexico in lakes and wetlands (Aladro et al., 2007; Bobrov et al., 2013).

\section{Arcella megastoma Penard, 1902}

Autogenous yellow or brown test. Circular in ventral view. Wide aperture with a small lip surrounded by small pores. Flattened in lateral view (Fig. 2H, Fig. 2I, Fig. 5D). The species was observed previously in Mexico in springs, lakes and wetlands (López-Ochoterena \& Madrazo-Garibay, 1979; Bobrov et al., 2013).

\section{Arcella vulgaris Ehrenberg, 1830}

Autogenous yellow or brown test. Circular in ventral view, with an invaginated, circular aperture bordered by a small lip. Smooth surface test with plates and numerous fine pores between them. Hemispherical in lateral view, often with a basal collar (Fig. 2J, Fig. 5E). This species has a broad distribution in Mexico and has been observed in springs, lakes, semipermanent freshwater bodies, cave pools, cenotes, drinking water, wastewater, wetlands, rice paddies and soil (Osorio-Tafall, 1943; LópezOchoterena \& Roure-Cane, 1970; Rico-Ferrat \& López-Ochoterena, 1976; Tomasini-Ortiz \& López-Ochoterena, 1979; Serrano-Limón \& López-Ochoterena, 1992; Aladro et al., 2007; Tiscareño, 2008; van Hengstum et al., 2008; Bobrov et al., 2013).

\section{Argynnia triangulata Deflandre, 1936}

Xenogenic brown test. Trapezoid shape in lateral view, with the fundus larger than the aperture. Aperture terminal and circular. Test surface smooth with regular and flattened mineral aggregates (Fig. 2K). This is the first record of this species in Mexico.

\section{Centropyxis aculeata \\ Ehrenberg, 1832 var. aculeata}

Xenogenous yellow or brown test. Ovoid or circular and depressed in ventral view, with 4-10 posterolateral spines. Aperture subterminal, circular or oval and invaginated. Capshaped in lateral view and tapers toward the aperture. Test surface is smooth only around the aperture and is covered near the spines and on the dorsal surface with mineral grains or diatom frustules (Fig. 2L, Fig. 2M, Fig. 2N, Fig. 5F, Fig 5G). This variety was observed previously in Mexican cenotes (van Hengstum et al., 2008). 


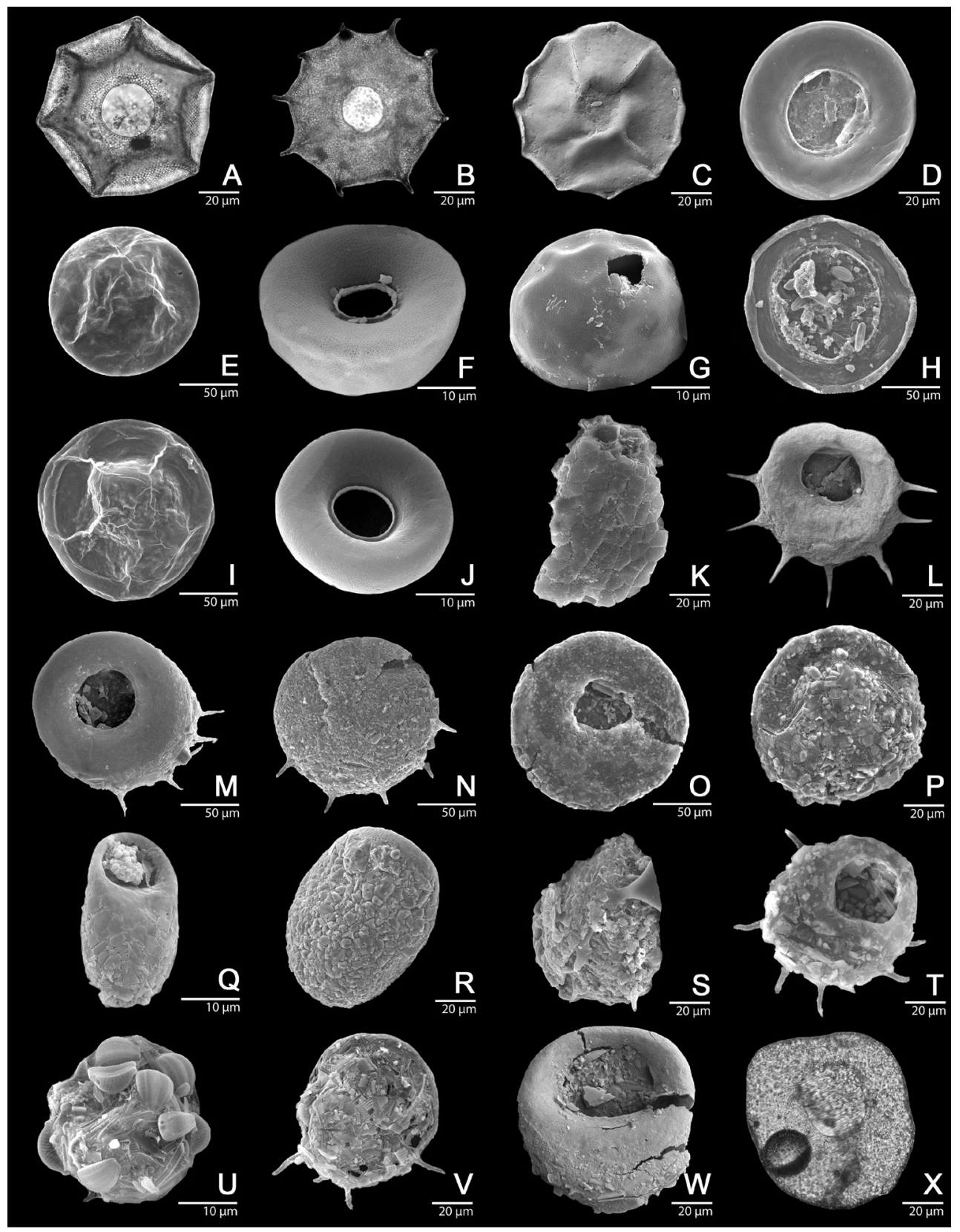

Fig. 2. Micrographs of tests from the study lakes (A) - (B) light microscopy; (C) - (W) scanning electron microscopy. Arcella conica (A) ventral view from lake No. 7; Arcella dentata (B) ventral view from lake No. 28, (C) dorsal view from lake No. 2; Arcella discoides (D) ventral view from lake No. 9, (E) dorsal view from lake No. 1; Arcella gibbosa from lake No. 5 (F) ventral view, (G) dorsal view; Arcella megastoma from lake No. 23 (H) ventral view, (I) dorsal view; Arcella vulgaris (J) ventral view from lake No. 2; Argynnia triangulata $(\mathbf{K})$ lateral view from lake No. 20; Centropyxis aculeata var. aculeata (L) ventral view from lake No. 10, (M) ventral view from lake No. 2, (N) dorsal view from lake No. 11; Centropyxis aculeata var. discoides from lake No. $28(\mathbf{O})$ ventral view, (P) dorsal view; Centropyxis constricta var. aerophila $(\mathbf{Q})$ ventral view from lake No. 28, (R) dorsal view from lake No. 17; Centropyxis constricta var. constricta from lake No. 29 (S) lateral view; Centropyxis constricta var. spinosa from lake No. 3 (T) ventral view, (U) dorsal view, (V) dorsal view form lake No. 28; Centropyxis deflandrei $(\mathbf{W})$ ventral view, from lake No. 2; Light microscopy, Centropyxis ecornis (X) ventral view from lake No. 10. 


\section{Centropyxis aculeata}

Ehrenberg, 1832 var. discoides

Xenogenous yellow or brown test. Ovoid or circular, depressed in ventral view and without spines. Aperture sub-terminal, circular or oval and invaginated. Main body hemispherical in lateral view with pronounced tapering toward the aperture. Test surface is rough and often covered with mineral grains (Fig. 2O, Fig. 2P, Fig. $5 \mathrm{H}$ ). This variety was observed previously in Mexican cenotes (van Hengstum et al., 2008).

\section{Centropyxis constricta}

Ehrenberg, 1843 var. aerophila

Xenogenous yellow or brown test. Ovoid in ventral view. Aperture sub-terminal, circular or ovoid, invaginated and with a semi-circular rim. Spherical in lateral view and slightly flattened towards the aperture. The test surface is generally rough with mineral grains or diatom frustules and smooth only in a small region around the aperture (Fig. 2Q, Fig. 2R, Fig. 5I). This variety was observed previously in Mexican cenotes (van Hengstum et al., 2008).

\section{Centropyxis constricta}

Ehrenberg, 1843 var. constricta

Xenogenous yellow or brown test. Ovoid in ventral view, with 2 or 3 spines on the fundus. The aperture is sub-terminal, ovoid, invaginated and with a semi-circular rim. Spherical in lateral view and slightly flattened towards the aperture. The test surface is rough with mineral grains (Fig. 2S). This variety was observed previously in Mexican cenotes (van Hengstum et al., 2008).

\section{Centropyxis constricta}

Ehrenberg, 1843 var. spinosa

Xenogenous yellow or brown test. Ovoid in ventral view, with 2-6 spines on the fundus. Aperture sub-terminal, ovoid, invaginated and with a semi-circular rim. Spherical in lateral view and flattened towards the aperture. The test surface is rough with mineral grains or diatom frustules (Fig. 2T, Fig. 2U, Fig. 2V). This is the first record of this variety in Mexico.

\section{Centropyxis deflandrei Rampi, 1950}

Xenogenous brown test. Circular in ventral view and laterally depressed. Aperture ovoid to circular and sub-terminal, almost half the size of the test. Without spines. Test surface with mineral grains or diatom frustules (Fig. $2 \mathrm{~W})$. This is the first record of this species in Mexico. A similar species (Centropyxis cf. deflandrei), however, was reported previously in soils and wetlands (Bobrov et al., 2013).

\section{Centropyxis ecornis Ehrenberg, 1841}

Xenogenous circular to square brown test. Circular in ventral view, flattened in lateral view. Large aperture, almost quadrate and sub-terminal, without spines. Test surface with mineral grains (Fig. 2X). Species previously observed in Mexico in lakes, waterfalls, wetlands and soil (Laminger, 1973; LópezOchoterena \& Madrazo-Garibay, 1979; Bobrov et al., 2013).

\section{Cucurbitella tricuspis Carter, 1856}

Xenogenous brown test. Spherical to vaseshaped in lateral view, with a terminal, circular and crenulated aperture. Often with a thin lip surrounded by a 5-11-lobe collar. Test surface with mineral grains or diatom frustules (Fig. 3A, Fig. 3B, Fig. 5J, Fig. 5K, Fig. 5L). This is the first record of this species in Mexico.

\section{Cyclopyxis kahli Deflandre, 1929}

Xenogenous brown test. Circular in ventral view, with a circular and central aperture. Hemispherical in lateral view. Aperture surface slightly invaginated and smooth. The dorsal surface is rough with mineral grains (Fig. 3C, Fig. 3D). Species previously observed in Mexico, in lakes, mosses, wetlands and soils (Golemansky, 1967; Bonnet, 1977; Bobrov \& Krasil'nikov, 2011; Bobrov et al., 2013). 


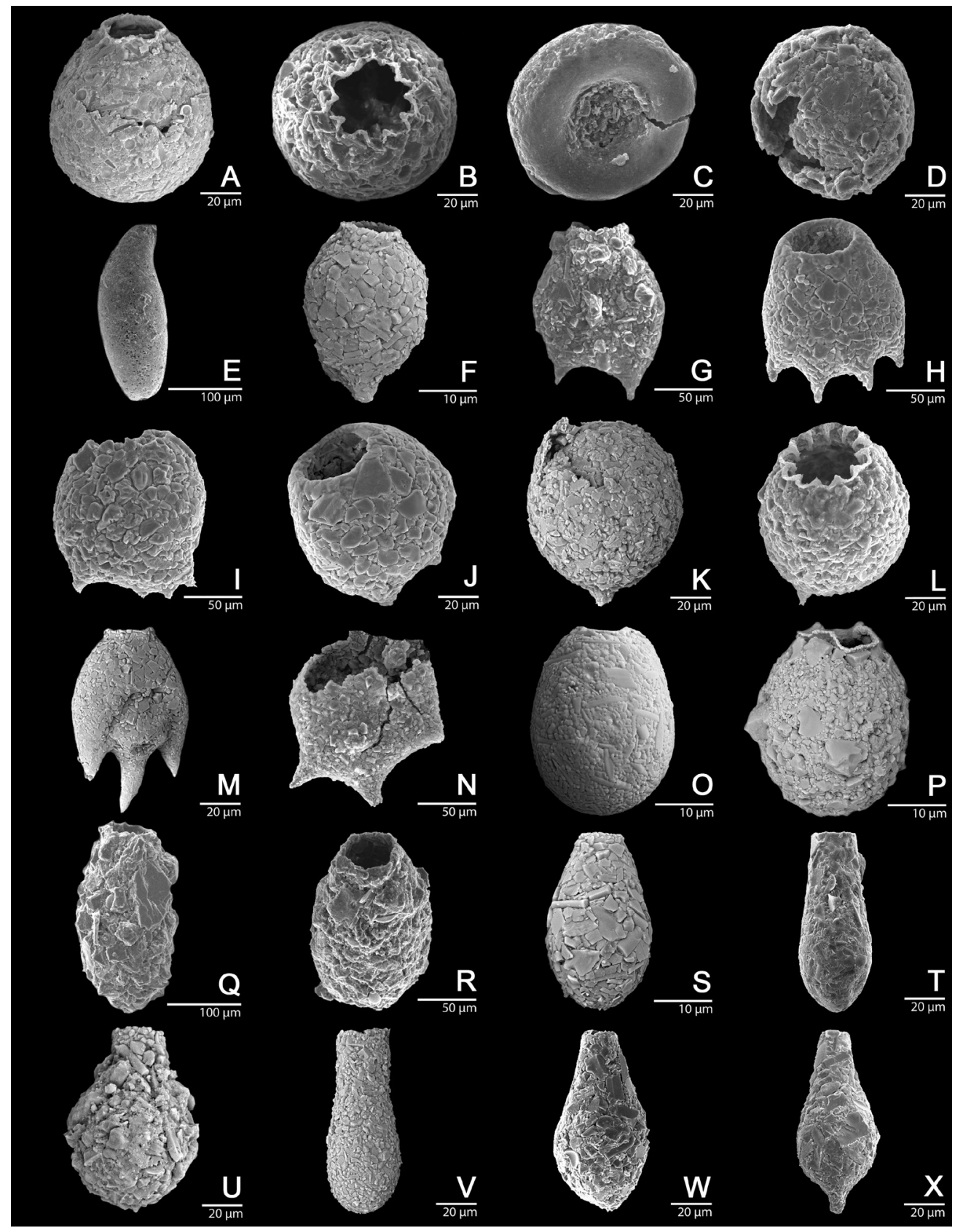

Fig. 3. Micrographs of tests from the study lakes, scanning electron microscopy. Cucurbitella tricuspis (A) lateral view from lake No. 29, (B) ventral view from lake No. 1; Cyclopyxis kahli from lake No. 20 (C) ventral view, (D) dorsal view; Cyphoderia ampulla (E) lateral view from lake No. 20; Difflugia labiosa (F) lateral view from lake No. 5; Difflugia distenda (G) lateral view from lake No. 29; Difflugia bidens from lake No. 8 (H) lateral view, (I) dorsal view, (J) ventral view; Difflugia corona $(\mathbf{K})$ lateral view from lake No. 5, (L) ventral view from lake No. 17; Difflugia fragosa lateral view (M) from lake No. 5, (N) from lake No. 19; Difflugia gramen (O) lateral view from lake No. 9, (P) lateral view from lake No. 27; Difflugia oblonga var. bryophila from lake No. 20 (Q) lateral view, (R) ventral view; Difflugia oblonga var. lanceolata (S) lateral view from lake No. 5; Difflugia oblonga var. linearis (T) lateral view from lake No. 23; Difflugia oblonga var. oblonga lateral view (U) from lake No. 29, (V) from lake No. 12; Difflugia oblonga var. spinosa (W) - (X) lateral view from lake No. 25. 


\section{Cyphoderia ampulla Ehrenberg, 1840}

Autogenous brown test. Ovoid in lateral view, with round base and tapered near the aperture, forming a short, retort-shaped neck. Aperture terminal and circular. The surface test is composed of oval plates on an organic matrix (Fig. 3E, Fig. 5M). Species previously observed in a lake in Mexico (Bobrov et al., 2013).

\section{Difflugia labiosa \\ (Leidy, 1874) Penard, 1902}

Xenogenous brown test. Pyriform in lateral view. Fundus tapers slightly to a rounded eccentric apex, with a thin collar surrounding the aperture, which is terminal and circular. Small mineral grains on the surface test (Fig. $3 \mathrm{~F})$. This is the first record of this species in Mexico.

\section{Difflugia distenda}

Gauthier-Lièvre and Thomas, 1958

Xenogenous test with colorful mineral grains. Almost spherical, with two symmetrical hornlike spines at the fundus. Thin collar with small mineral grains and terminal aperture (Fig. 3G). This is the first record of this species in Mexico.

\section{Difflugia bidens Penard, 1902}

Xenogenous brown test. Ovoid and laterally compressed, with 2-5 small spines at the fundus. Aperture terminal, circular and large, without a collar. Some spines can be bifurcated. Test surface with relatively smooth mineral grains (Fig. 3H, Fig. 3I, Fig. 3J). This is the first record of this species in Mexico.

\section{Difflugia corona Wallich, 1864}

Xenogenous brown test. Ovoid to spherical in lateral view, with 1-4 spines at the fundus. Some spines can be bifurcated. Aperture terminal, circular and surrounded by a collar with 8 -12 lobes composed of small particles cemented together. Test surface formed of small mineral grains or fragments of diatom frustules (Fig. 3K, Fig. 3L, Fig. 5N). Species previously observed in Mexico in lakes and in a reservoir (López-Ochoterena \& Roure-Cane, 1970; Serrano-Limón \& López-Ochoterena, 1992; Silva-Briano et al., 2007).

\section{Difflugia fragosa Hempel, 1898}

Xenogenous, yellow or brown test. Elongated, with 3-5 large projections running from the middle of the test to the fundus, forming wide spines or tubercles. Aperture terminal and circular, with a thin collar. Test surface formed of small mineral grains (Fig. 3M, Fig. 3N). This is the first record of this species in Mexico.

\section{Difflugia gramen Penard, 1902}

Xenogenous, yellow or brown test. Test ovoid or spherical in lateral view. Aperture terminal and lobed, in general with 3 lobules and a thin lip made of small particles cemented together. Test surface formed of small mineral grains (Fig. 3O, Fig. 3P, Fig. 5O). Species previously observed in Mexico in lakes, wetlands and wastewater (López-Ochoterena \& RoureCane, 1970; Rico-Ferrat \& López-Ochoterena, 1976; Serrano-Limón \& López-Ochoterena, 1992; Bobrov et al., 2013).

\section{Difflugia oblonga \\ Ehrenberg, 1832 var. bryophila}

Xenogenous, brown test. Pyriform in lateral view, with a conical fundus. Aperture terminal and circular, formed by mineral grains cemented together, without a collar. Test made of coarse sand grains (Fig. 3Q, Fig. 3R, Fig. 5P). This is the first record of this variety in Mexico.

\section{Difflugia oblonga \\ Ehrenberg, 1832 var. glans}

Xenogenous, brown test. Ovoid in lateral view, with a rounded fundus. Aperture terminal, large, circular and formed by mineral grains cemented together, without a collar. Test made of coarse sand grains. This is the first record of this variety in Mexico. 


\section{Difflugia oblonga}

Ehrenberg, 1832 var. lanceolata

Xenogenous, brown test. Pyriform in lateral view, with a rounded fundus. Aperture terminal, circular and formed by mineral grains cemented together, without collar. Test made of small mineral grains (Fig. 3S). This is the first record of this variety in Mexico.

\section{Difflugia oblonga}

Ehrenberg, 1832 var. linearis

Xenogenous, brown test. Ovoid and elongated in lateral view, with a conical fundus. Aperture terminal and circular, without a collar, formed by mineral grains cemented together. Test made of small mineral grains (Fig. 3T). This is the first record of this variety in Mexico.

\section{Difflugia oblonga \\ Ehrenberg, 1832 var. oblonga}

Xenogenous, brown test. Pyriform and elongated in lateral view, with a large neck and rounded fundus. Aperture terminal, circular and formed by mineral grains cemented together. Test composed of angular sand grains and diatom frustules (Fig. 3U, Fig. 3V). This is the first record of this variety in Mexico.

\section{Difflugia oblonga}

Ehrenberg, 1832 var. spinosa

Xenogenous, brown test. Pyriform and elongated in lateral view, with a short or long neck and a spine on the fundus. Aperture terminal, circular and formed by mineral grains cemented together. Test composed of angular sand grains and diatom frustules (Fig. 3W, Fig. 3X). This is the first record of this variety in Mexico.

\section{Difflugia oblonga}

Ehrenberg, 1832 var. tenuis

Xenogenous, brown test. Pyriform and elongated in lateral view, without a neck and a truncated fundus. Aperture terminal and circular, formed by mineral grains cemented together. Test composed of angular sand grains and diatom frustules (Fig. 4A, Fig. 4B). This is the first record of this variety in Mexico.

\section{Difflugia protaeiformis \\ Lamarck, 1816 var. acuminata}

Xenogenous, hyaline to brown test. Cylindrical in lateral view, with slight tapering near the aperture and a dorsal spine sometimes very large and slightly curved. Aperture terminal and circular, with a smooth outline. Test made of mixed-size mineral grains or diatom frustules (Fig. 4C, Fig. 4D, Fig. 4E, Fig. 5Q). This is the first record of this variety in Mexico.

\section{Difflugia protaeiformis \\ Lamarck, 1816 var. amphoralis}

Xenogenous, yellow to brown. Elongated test, almost biconical in lateral view, narrowing toward the aperture and with a large projection in the dorsal region that forms one or two wide spines. Aperture terminal and circular, with a smooth outline and a short collar. Test made of coarse grains (Fig. 4F, Fig. 4G). This is the first record of this variety in Mexico.

\section{Difflugia protaeiformis \\ Lamarck, 1816 var. claviformis}

Xenogenous, opaque test. Cylindrical in lateral view, slightly tapering near the aperture and with a wide spine in the dorsal region. Aperture terminal and circular, with a smooth outline. Test made of coarse grains (Fig. 4H). This is the first record of this variety in Mexico.

\section{Difflugia urceolata \\ Carter, 1864 var. elongata}

Xenogenous, brown and opaque test. Ovoid in lateral view, with a rim sometimes pronounced and curved. Some specimens can have wide spine-like protuberances. Aperture large, terminal and circular, with a relatively smooth outline. Test made of mixed-size grains or diatom frustules (Fig. 4I, Fig. 4J, Fig. 5T). This is the first record of this variety in Mexico. 

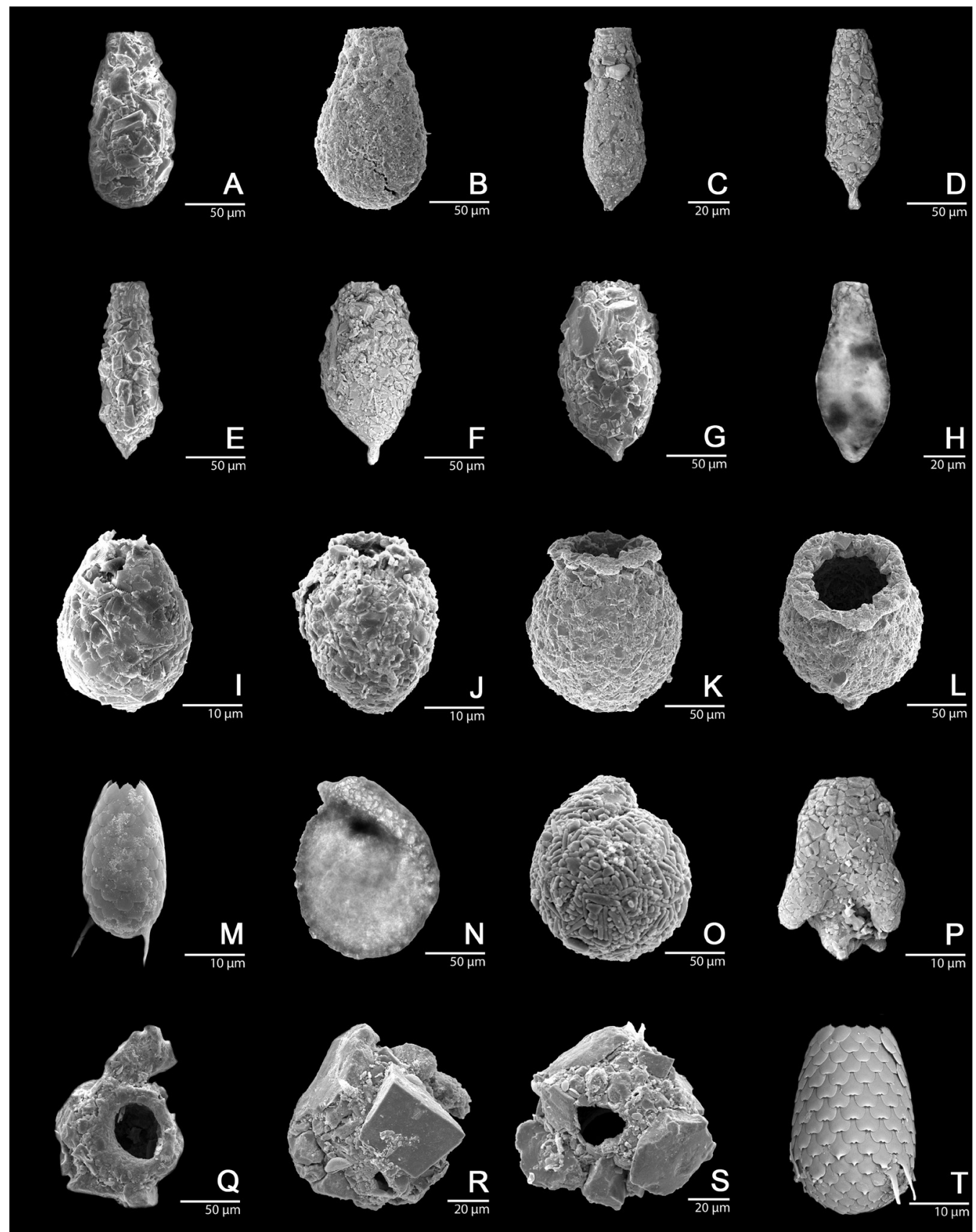

Fig. 4. Micrographs of tests from the study lakes (A) - (G), (I) - (M), (O) - (T) scanning electron microscopy; (H) and (N) light microscopy. Difflugia oblonga var. tenuis lateral view (A) from lake No. 28, (B) from lake No. 10; Difflugia protaeiformis var. acuminata lateral view (C) from lake No. 29, (D) from lake No. 27 (E) from lake No. 1; Difflugia protaeiformis var. amphoralis lateral view (F) from lake No. 29, (G) from lake No. 28; Difflugia protaeiformis var. claviformis $\mathbf{( H )}$ lateral view from lake No. 10; Difflugia urceolata var. elongata (I) lateral view from lake No. 7, (J) ventral view from lake No. 18; Difflugia urceolata var. urceolata from lake No. 17 (K) lateral view, (L) ventral view; Euglypha acanthophora $(\mathbf{M})$ lateral view from lake No. 28; Lesquereusia modesta $(\mathbf{N})$ lateral view from lake No. 14; Lesquereusia spiralis $(\mathbf{O})$ lateral view from lake No. 1; Pentagonia maroccana $(\mathbf{P})$ lateral view from lake No. 5; Pseudodifflugia fulva from lake No. 3 (Q) ventral view, (R) - (S) dorsal view; Scutiglypha cabrolae (T) lateral view from lake No. 22. 


\section{Difflugia urceolata \\ Carter, 1864 var. urceolata}

Xenogenous, brown and opaque test. Spherical in lateral view, with a pronounced rim that curves back on itself. Occasional wide, spine-like protuberances. Aperture large, terminal and circular with a relatively smooth outline. Test made of mixed-sized grains or diatom frustules (Fig. 4K, Fig. 4L). This is the first record of this variety in Mexico.

\section{Euglypha acanthophora Ehrenberg, 1814}

Autogenous hyaline test. Ovoid in lateral view, with a rounded fundus. Aperture terminal and circular. Test composed of oval to hexagonal plates. Those forming the aperture display a serrated, V-shaped edge, with 5-7 teeth bordering each of these margins. Some dorsal-region plates project dorsally like spines, and can be up to three times the size of the plates themselves (Fig. 4M, Fig. 5R). Species previously observed in Mexico in lakes and wetlands (Aladro et al., 2007; Bobrov et al., 2013).

\section{Lesquereusia modesta Rhumbler, 1895}

Autogenous, hyaline to brown. Spherical in lateral view, with an asymmetrical neck, and laterally, slightly narrow. Aperture terminal and circular. Test surface composed of small mineral grains (Fig. 4N). Species previously observed in Mexican high-mountain lakes (Laminger, 1973).

\section{Lesquereusia spiralis Ehrenberg, 1840}

Autogenous, brown test. Spherical in lateral view, with an asymmetrical neck and laterally, slightly narrow. Spine-like dorsal projection. Aperture terminal and circular. Test surface composed of numerous curved rods (Fig. 4O, Fig. 5S). This is the first record of this species in Mexico.

\section{Pentagonia maroccana}

Gauthier-Lièvre and Thomas, 1958

Xenogenous brown test. Elongated in lateral view, with four short, wide, spine-like projections on fundus. Short collar and terminal aperture. Surface with small mineral grains (Fig. 4P). This is the first record of this species in Mexico.

\section{Pseudodifflugia fulva Archer, 1870}

Xenogenous brown test. Spherical in lateral view, with a very irregular shape because of the large agglutinated particles. Aperture circular, large and smooth in ventral view (Fig. 4Q, Fig. 4R, Fig. 4S). This is the first record of this species in Mexico.

\section{Scutiglypha cabrolae \\ Smet and Gibson, 2009}

Autogenous test. Plates of different shape depending on the row level. Plates in row around aperture with a triangular edge forming a serrated margin. The opposite end of the plate is semi-circular in shape. Rows of plates increasingly distant from the aperture have decreasingly rounded upper edges, culminating in generally small, but sometimes longer and wider spines in the rows at the fundus (Fig. 4T). This is the first record of this species in Mexico.

Taxa distribution in lakes: The greatest number of taxa found in a single lake (No. 4) was 18 , and only one lake (No. 6) had no individuals. (Species richness in each lake was tabulated in Appendix I). On average, we recorded eight taxa in each lake. The most-observed taxon was Centropyxis aculeata var. aculeata, observed in 22 of the 29 lakes, followed by Arcella discoides, recorded in 17 lakes. In contrast, eight species were recorded in only one lake (Arcella conica, Centropyxis deflandrei, C. ecornis, Cyphoderia ampulla, Difflugia distenda, Lesquereusia spiralis, Pseudodifflugia fulva and Scutiglypha cabrolae). The most similar lakes in terms of species composition were lakes No. 1 and 19, which shared 10 species. Specific richness of testate amoebae in the lakes does not seem to be associated with water depth or lake altitude, so other environmental variables should be explored. 


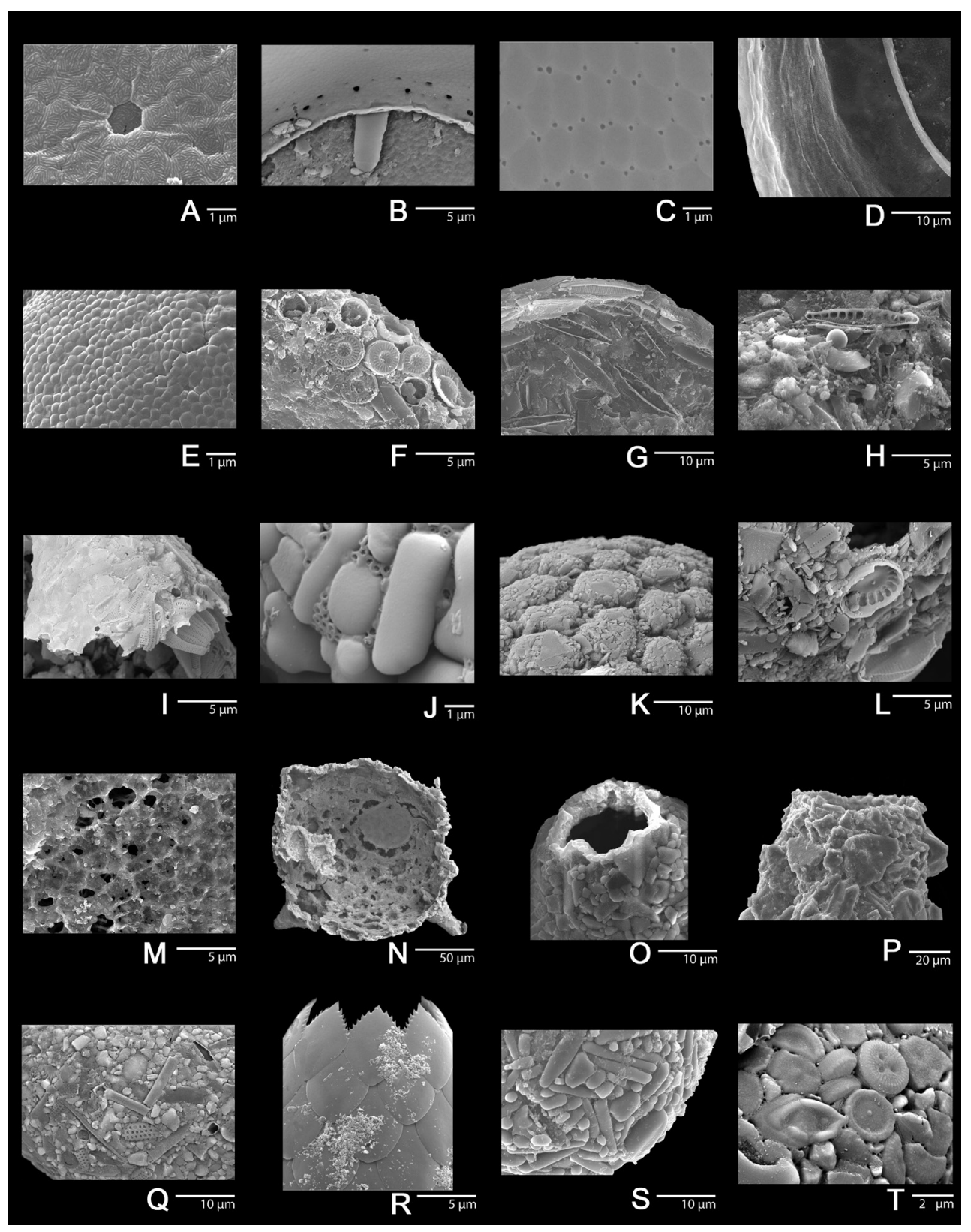

Fig. 5. Micrographs of tests from the study lakes, scanning electron microscopy. Arcella dentata (A) from lake No. 2, detail of plates; Arcella discoides (B) from lake No. 10, detail of the border of the aperture and its pores; Arcella gibbosa (C) from lake No. 5, detail of plates and pores; Arcella megastoma (D) from lake No. 3, border of the test and the aperture; Arcella vulgaris (E) from lake No. 2, detail of plates; Centropyxis aculeata var. aculeata, diatoms of the test (F) from lake No. 9, (G) from lake No. 11; Centropyxis aculeata var. discoides $(\mathbf{H})$ from lake No. 28, diatoms of the test; Centropyxis constricta var. aerophila (I) from lake No. 24, material of test; Cucurbitella tricuspis (J) from lake No. 9, particles of test and cement matrix, (K) - (L) from lake No. 10, agglutinated material; Cyphoderia ampulla (M) from lake No. 20, detail of plates; Difflugia corona (N) from lake No. 10, interior structure of test; Difflugia gramen (O) from lake No. 15, detail of the aperture; Difflugia oblonga var. bryophila (P) from lake No. 20, detail of the aperture; Difflugia protaeiformis var. acuminata $(\mathbf{Q})$ from lake No. 27, agglutinated material; Euglypha acanthophora $(\mathbf{R})$ from lake No. 28, detail of the aperture; Lesquereusia spiralis (S) from lake No. 1, detail of plates; Difflugia urceolata var. elongata (T) from lake No. 29, diatoms of the test. 


\section{DISCUSSION}

There have been few studies on testate amoebae in Mexican lakes. Only recently these protozoa have become the subject of study in Mexico. The focus, however, has been more on soil organisms than on those in aquatic environments. Taxonomic work on testate amoebae in the Neotropics is difficult, owing to the lack of good descriptions or illustrations that could facilitate species recognition. This study provides new biogeographic information and presents the first SEM photographs of almost all species recorded in Mexico.

Recent schemes of classification are based more on the type of pseudopods that testate amoebae possess (Meisterfeld, 2002a, 2002b) or molecular data (Nikolaev et al. 2005). In fact, new molecular studies identified cryptic species and showed the polyphyletic nature of the group (Tekle et al., 2008; Oliverio, Lahr, Nguyen, \& Katz, 2014). The characters used for identification in the majority of descriptive and ecologic studies were, however, mainly based on test morphology. Because testate amoebae have few morphologic characters, biometric analysis is a critical aspect of phenotypic description (Bobrov \& Mazei, 2004).

Among our specimens, we saw a large size range for the genus Arcella, but the ratio between the test diameter and aperture distinguishes well the different species, without the need for species varieties. Only A. megastoma has great size plasticity. In the case of the genus Centropyxis, all varieties are well defined by the relation between length and width of the test, and the aperture size grows as the test size increases. C. constricta var. aerophila shows large size variation, which may be a consequence of the fact that measured specimens came from different lakes. This is also true for three varieties of Difflugia oblonga ( $D$. oblonga var. tenuis, D. oblonga var. oblonga and $D$. oblonga var. spinosa) which show great variability and the specimens came from different lakes as well. In contrast, D. oblonga var. bryophila, D. oblonga var. linearis, and both varieties of $D$. protaeiformis and D. urceolata, display differences in size that, in conjunction with shape differences, support their distinction as different varieties (Appendix II).

Bobrov and Mazei (2004) proposed that polymorphic populations could reflect habitat heterogeneity, thus broad size ranges in our study taxa could be consequence of different collection depths. In samples from shallow lakes, this could also reflect the large number of microhabitats provided by aquatic vegetation.

We compared our morphological data with those from other studies and noticed that Difflugia gramen was smaller in our lakes (Table 2) than in lakes of the study by Lahr and Lopes (2006) (average test length $=108 \mu \mathrm{m}$, average test width $=92.3 \mu \mathrm{m}$, average aperture diameter $=38 \mu \mathrm{m}$ ), but very similar to the size of specimens reported by Bobrov and Mazei (2004) (average test length $=68.8 \mu \mathrm{m}$, average test width $=51.5 \mu \mathrm{m}$, average aperture diameter $=23.3 \mu \mathrm{m})$. Difflugia corona individuals also show substantial size variability. Our specimens were smaller than those reported by Lahr and Lopes (2006): (average test length $=192.1$ $\mu \mathrm{m}$, average test width $=189.8 \mu \mathrm{m}$, average aperture diameter $=91.6 \mu \mathrm{m}$; Bobrov $\&$ Mazei, 2004: average test length $=166.1 \mu \mathrm{m}$, average test width $=159.9 \mu \mathrm{m}$, average aperture diameter $=67.9 \mu \mathrm{m})$. Again, this size variability may reflect differences among populations. Thus, it may be appropriate to assign a variety name to our specimens if environmental conditions are responsible for determining their morphometry.

Some species found in this study, such as Arcella dentata, A. discoides, A. gibbosa, A. megastoma, Centropyxis aculeata var. aculeata, C. aculeata var. discoides, C. constricta var. aerophila, C. constricta var. constricta, Cyphoderia ampulla, Difflugia corona and Lesquereusia modesta, had been recorded previously in Mexico, associated with a variety of freshwater habitats, including springs, lakes, rivers, cenotes, cave pools, drinking troughs and wastewater. Others, such as Arcella vulgaris and Cyclopyxis kahli, were found in mosses and soils as well. Centropyxis aculeata var. aculeata, the taxon found in nearly two-thirds of our sample lakes, was previously reported 
only in a recent work (van Hengstum et al., 2008). This may be a consequence of the fact that researchers in several previous studies in Mexico identified individuals only to species level, whereas in recent studies, identifications were made to the variety level.

Lakes of the same region displayed different species richness. This could indicate differences in environmental conditions, underscoring the need for detailed studies that include collection of data on physical and chemical variables in the lakes.

In a brief review of testate rhizopod studies in lakes around the world, we noted that the species assemblages we report from Central Mexico resemble most closely assemblages from Neartic water bodies close to Lake Ontario, and near other Canadian lakes (Patterson, MacKinnon, Scott, \& Medioli, 1985; Patterson, Barker, \& Burbidge, 1996; Reinhardt, Dalby, Kumar, \& Patterson, 1998; Kumar \& Patterson, 2000; Torigai, Schröder-Adams, \& Burbidge, 2000; Roe, Patterson, \& Swindles, 2009; Roe \& Patterson 2014). In those studies, the composition and abundance of species generally indicated mesotrophic conditions and pollution, suggesting it will be important to gather such information on Mexican lakes to see if testate amoebae can serve as bioindicators of these variables in those water bodies.

The high number of new records provided by this study shows the scarcity of information regarding the diversity of testate amoebae in lakes of Mexico. The morphometric data suggest that new varieties could be described in lakes throughout the center of the country. Only through a comprehensive species characterization will it be possible to carry out ecological and palaeoecological studies in the future.

\section{ACKNOWLEDGMENTS}

We thank the Posgrado de Ciencias Biológicas, UNAM (CVU 294479 CONACYT). This research was funded by grants from CONACYT (167 621), NSF (0 902 864) and the Instituto de Geología, Universidad Nacional Autónoma de México, for financial support. We thank Margarita Caballero and Edyta Zawisza (Instituto de Geofísica, UNAM), Alexander Correa-Metrio and Esperanza Torres (Instituto de Geología, UNAM) for field help. Special thanks to Alejandro Angeles, Mariela Esquivel, Jacqueline Jasso, Antonio Guerra and Manuel Valle for help in laboratory. We thank Natalia Hoyos for the map elaboration, Enrique Lara and Anatoly Bobrov for help with identifications of some species and Mark Brenner for editorial assistance.

\section{RESUMEN}

Amebas testadas (Amebozoa, Arcellinida) en lagos tropicales del centro de México. Las amebas testadas son organismos eucariontes unicelulares frecuentes en ecosistemas acuáticos. A pesar de su importante rol en estos ecosistemas, y su potencial como bioindicadores y paleoindicadores, son poco estudiados en México. Los principales objetivos de este estudio fueron: 1) incrementar el conocimiento de las amebas testadas en lagos tropicales de México y 2) crear un catálogo de microfotografías electrónicas de barrido de alta calidad que pueda ser usado en futuros estudios ecológicos y paleoambientales. Recolectamos muestras de sedimentos superficiales de 29 lagos, ubicados en la Faja Volcánica Transmexicana, una vez en cada lago durante junio y octubre 2011 y marzo 2013. Los sedimentos se recolectaron con una draga tipo Ekman y se preservaron en etanol anhidro. Las sub-muestras se observaron al microscopio estereoscópico. Para cada especie fueron registrados sus datos morfométricos. En testas circulares fueron medidos el diámetro y la apertura total. En testas irregulares fueron medidos el largo y ancho de la apertura y el tamaño de toda la testa. Si un ejemplar poseía espinas, se seleccionó una al azar y se midió su longitud. El ejemplar mejor preservado de cada taxón fue fotografiado con un microscopio óptico y en microscopio electrónico de barrido (MEB). Encontramos 41 taxa de amebas testadas pertenecientes a los géneros Arcella, Argynnia, Centropyxis, Cucurbitella, Cyclopyxis, Cyphoderia, Difflugia, Euglypha, Lesquereusia, Pentagonia, Pseudodifflugia y Scutiglypha. Se registraron 12 especies y 13 variedades que no estaban reportadas previamente en México. El número promedio de taxa registrados en cada lago fue de ocho, y la riqueza taxonómica mayor fue de 18. El taxón encontrado en el mayor número de lagos fue Centropyxis aculeata var. aculeata. La riqueza taxonómica compartida por lagos de la misma región no fue muy similar. Esto puede reflejar diferencias en las condiciones ambientales, evidenciando la necesidad de elaborar estudios más detallados que incluyan la recolección de datos de las variables físico-químicas en los lagos. Nuestros resultados resaltan la necesidad de estudios posteriores acerca de los patrones de distribución y la ecología de las amebas testadas lacustres. 
Palabras clave: protozoos, ameboides, tecamebas, taxonomía, biometría.

\section{REFERENCES}

Aladro, M. A., Reyes, M., Olvera, F., \& Robles, M. N. (2007). Ciliados y otros protozoos. In A. Lot (Ed.), Guía Ilustrada de la Cantera Oriente: Caracterización Ambiental e Inventario Biológico (pp. 97-122). Distrito Federal, México: Coordinación de la Investigación Científica, Secretaría Ejecutiva de la Reserva Ecológica del Pedregal de San Ángel de Ciudad Universitaria, UNAM.

Bobrov, A., \& Mazei, Y. (2004). Morphological variability of testate amoebae (Rhizopoda: Testacealobosea: Testaceafilosea) in natural populations. Acta Protozoologica, 43, 133-146.

Bobrov, A. A., \& Krasil'nikov, P. A. (2011). Testate amoebas of pine forests in Mexico. Biology Bulletin, $4,470-476$.

Bobrov, A., Krasil'nikov, P., \& García-Calderón, N. E. (2013). Biogeography of testate amoebae in the soils of Mexico. Biodiversity and Conservation, 22, 2837-2855.

Bonnet, L. (1977). Faunistique et biogêographie des Thêcamoebiens. I. Thécamoebiens des sols du Mexique. Extrait du Bulletin de la Société D'Histoire Naturelle de Toulouse, 113(1-2), 1-5.

Charman, D. J., Hendon, D., \& Woodland, W. (2000). The Identification of Testate Amoebae (Protozoa: Rhizopoda) in Peats (Quaternary Research Association Technical Guide No. 9). London, UK: Quaternary Research Association.

Ellison, R. L., \& Ogden, C. G. (1987). A guide to the study and identification of fossil testate amoebae in Quaternary lake sediments. Internationale Revue der Gesamten Hydrobiologie, 72(5), 639-652.

Escobar, J., Martínez, J. I., \& Parra, L. N. (2005). Thecamoebians (Testaceous rhizopods) from a tropical lake: La Fe Reservoir, Antioquia, Colombia. Caldasia, 27, 293-298.

Escobar, J., Brenner, M., Whitmore, T. J., Kenney, W. F., \& Curtis, J. H. (2008). Ecology of testate amoebae (thecamoebians) in subtropical Florida lakes. Journal of Paleolimnology, 40, 715-731.

Gallegos-Neyra, E. M., Lugo-Vázquez, A., Calderón-Vega, A., Sánchez-Rodríguez, M. R., \& Mayén-Estrada, R. (2014). Biodiversidad de protistas amébidos de vida libre en México. Revista Mexicana de Biodiversidad, $85,10-25$.

Golemansky, V. (1967). Tecamebianos muscicolas (Rhizopoda, Testacea) de México. Revista de la Sociedad Mexicana de Historia Natural, 28, 73-78.
Hoffmann, A., Palacios, V. J. G., \& Morales-Malacara, J. B. (1986). Manual de Bioespeleología. Distrito Federal, México: Universidad Nacional Autónoma de México.

International Commission on Zoological Nomenclature (ICZN). (1999). International Code of Zoological Nomenclature. London, UK: The Natural History Museum.

Kumar, A., \& Dalby, A. P. (1998). Identification key for Holocene lacustrine arcellacean (thecamoebian) taxa. Palaeontologia Electronica, 1(1), 1-39.

Kumar, A., \& Patterson, R. T. (2000). Arcellaceans (Thecamoebians): New tools for monitoring long and short term changes in lake bottom acidity. Environmental Geology, 39, 689-697.

Lahr, J. G., \& Lopes, S. G. B. C. (2006). Morphology, biometry, ecology and biogeography of five species of Difflugia Leclerc, 1815 (Arcellinida: Difflugiidae), from Tiete River, Brazil. Acta Protozoologica, 45, 77-90.

Laminger, H. (1973). Die testaceen (Protozoa, Rhizopoda) einiger Hochgebirgsgewässer von Mexiko, Costa Rica und Guatemala. Internationale Revue der gesamten Hydrobiologie und Hydrographie, 58, 273-305.

Lee, J. J., Leedale, G., \& Bradbury, P. (2000). An Illustrated Guide to the Protozoa. Kansas, Misuri: Society of Protozoologists.

López-Ochoterena, E., \& Roure-Cane, M. T. (1970). Lista taxonómica comentada de protozoarios de vida libre de México. Revista de la Sociedad Mexicana de Historia Natural, 31, 23-68.

López-Ochoterena, E., \& Madrazo-Garibay, M. (1979). La contribución de C.G. Ehrenberg al conocimiento de los protozoarios de vida libre de México. Anales del Instituto de Biología, Universidad Nacional Autónoma de México, Serie Zoología, 50, 739-742.

Medioli, F. S., \& Scott, D. B. (1988). Lacustrine thecamoebians (mainly arcellaceans) as potential tools for palaeolimnological interpretations. Palaeogeography, Palaeoclimatology, Palaeoecology, 62, 361-386.

Medioli, F. S., Bonnet, L., Scott, D. B., \& Medioli, B. E. (2003). The The camoebian bibliography, 2nd edition. Palaeontologia Electronica, 6(5), 1-107.

Meisterfeld, R. (2002a). Order Arcellinida Kent, 1880. In J. J. Lee, G. Leedale, \& P. Bradbury (Eds.), An Illustrated Guide to the Protozoa (pp. 827-860). Kansas, USA: Society of Protozoologists.

Meisterfeld, R. (2002b). Testate amoebae with filopodia. In J. J. Lee, G. Leedale, \& P. Bradbury (Eds.), An Illustrated Guide to the Protozoa (pp. 1054-1084). Kansas, USA: Society of Protozoologists.

Mitchell, E. A. D., Charman, D. J., \& Warner, B. G. (2008). Testate amoebae analysis in ecological and paleoecological studies of wetlands: past, present and future. Biodiversity and Conservation, 17, 2115-2137. 
Neville, L. A., Christie, D. G., McCarthy, F. M. G., \& MacKinnon, M. D. (2010). Biogeographic variation in Thecamoebian (Testate amoeba) assemblages in lakes within various vegetation zones of Alberta, Canada. International Journal of Biodiversity and Conservation, 2, 215-224.

Nikolaev, S. I., Mitchell, E. A. D., Petrov, N. B., Berney, C., Fahrni, J., \& Pawlowski, J. (2005). The testate lobose amoebae (Order Arcellinida Kent, 1880) finally find their home within Amoebozoa. Protist, 156, 191-202.

Ogden, C. G., \& Hedley, H. (1980). An atlas of freshwater testate amoebae. Hampshire, UK: Oxford University Press.

Oliverio, A. M., Lahr, D. J. G., Nguyen, T., \& Katz, L. A. (2014). Cryptic diversity within morphospecies of testate amoebae (Amoebozoa: Arcellinida) in New England bogs and fens. Protist, 165, 196-207.

Osorio-Tafall, B. F. (1943). Observaciones sobre la fauna acuática de las cuevas de la región de Valles, San Luis Potosí (México). Revista de la Sociedad Mexicana de Historia Natural, 4, 43-71.

Patterson, R. T., \& Kumar, A. (2002). A review of current testate rhizopod (thecamoebian) research in Canada. Palaeogeography, Palaeoclimatology, Palaeoecology, 180, 225-251.

Patterson, R. T., MacKinnon, K. D., Scott, D. B., \& Medioli, F. S. (1985). Arcellaceans ("Thecamoebians") in small lakes of New Brunswick and Nova Scotia: modern distribution and Holocene stratigraphic changes. Journal of Foraminiferal Research, 15, 114-137.

Patterson, R. T., Barker, T., \& Burbidge, S. M. (1996). Arcellaceans (Thecamoebians) as proxies of arsenic and mercury contamination in northeastern Ontario Lakes. Journal of Foraminiferal Research, 26, 172-183.

Patterson, R. T., Roe, H. M., \& Swindles, G. T. (2012). Development of an Arcellacea (testate lobose amoebae) based transfer function for sedimentary phosphorus in lakes. Palaeogeography, Palaeoclimatology, Palaeoecology, 348-349, 32-44.

Reinhardt, E., Dalby, A. P., Kumar, A., \& Patterson, R. T. (1998). Arcellaceans as pollution indicators in mine tailing contaminated lakes near Cobalt, Ontario, Canada. Micropaleontology, 44, 131-148.

Rico-Ferrat, G. \& López-Ochoterena, E. (1976). Aspectos biológicos de los protozoarios de las aguas negras de la zona metropolitana de la ciudad de México. Revista de la Sociedad Mexicana de Historia Natural, 37, 221-228.
Roe, H. M., Patterson, R. T., \& Swindles, G. T. (2009). Controls on the contemporary distribution of lake thecamoebians (testate amoebae) within the Greater Toronto Area and their potential as water quality indicators. Journal of Paleolimnology, 43, 955-975.

Roe, H. M., \& Patterson, R. T. (2014). Arcellacea (Testate Amoebae) as bio-indicators of road salt contamination in lakes. Microbial Ecology, 68, 299-313.

Serrano-Limón, G. \& López-Ochoterena, E. (1992). Análisis sistemático del grupo de protozoarios estudiados del lago de Xochimilco, D.F. Revista de la Sociedad Mexicana de Historia Natural, 43, 63-69.

Silva-Briano, M., Martínez-Hernández, S. L., AdabacheOrtíz, A., Ventura-Juárez, J., Salinas, E., \& Quintanar, J. L. (2007). Ultrastructural analysis and identification of membrane proteins in the free-living amoeba Difflugia corona. Biocell, 31, 225-228.

Smith, H. G., Bobrov, A., \& Lara, E. (2008). Diversity and biogeography of testate amoebae. Biodiversity and Conservation, 17, 329-343.

Tekle, Y. I., Grant, J., Anderson, O. R., Nerad, T. A., Cole, J. C., Patterson, D. J., \& Katz, L. A. (2008). Phylogenetic placement of diverse amoebae inferred from multigene analyses and assessment of clade stability within 'Amoebozoa' upon removal of varying rate classes of SSU-rDNA. Molecular Phylogenetics and Evolution, 47, 339-352.

Tiscareño, R. S. (2008). Protozoos: Ciliados y sarcodinos. In V. H. Ávila, E. D. Melgarejo, \& A. C. Angón (Eds.), La Biodiversidad en Aguascalientes: Estudio de Estado Comisión Nacional para el Conocimiento y Uso de la Biodiversidad (pp. 118-121). Aguascalientes, México: Instituto del Medio Ambiente del Estado de Aguascalientes, Universidad Autónoma de Aguascalientes.

Tomasini-Ortiz P. \& López-Ochoterena, P. (1979). Análisis taxonómico de las especies de protozoarios encontradas en el agua potable de la ciudad de México, D.F. Revista Latinoamericana de Microbiología, 21, 147-151.

Torigai, K., Schröder-Adams, C. J., \& Burbidge, S. M. (2000). A variable lacustrine environment in Lake Winnipeg, Manitoba: Evidence from modern thecamoebian distribution. Journal of Paleolimnology, 23, 305-318.

van Hengstum, P. J., Reinhardt, E. G., Beddows, P. A., Huang, R. J., \& Gabriel, J. J. (2008). Thecamoebians (Testate amoebae) and foraminifera from three anchialine cenotes in Mexico: low salinity (1.5$4.5 \mathrm{psu}$ ) faunal transitions. Journal of Foraminiferal Research, 38, 305-317. 
